PoS $\quad$ PROCEEDINGS

\title{
Lattice calculation of the QGP viscosities - Present results and next project -
}

\section{Sunao Sakai*}

Faculty of Education, Art and Science, Yamagata University Kojirakawa 1-4-12, Yamagata, Yamagata 990-8560, Japan E-mail: sakaile.yamagata-u.ac.jp

\section{Atsushi Nakamura}

RIISE, Hiroshima University

Kagamiyama 1-7-1, HigashiHiroshima, Hiroshima 793-8521, Japan

E-mail: nakamura@riise.hiroshima-u.ac.jp

The shear and bulk viscosities of gluon plasma are calculated by accumulating a large amount of data for the Matsubara Green function $\left(G_{\beta}\left(t_{m}\right)\right)$ on isotropic $24^{3} \times 8$ and $16^{3} \times 8$ lattices. In the case of Iwasaki's improved action, $G_{\beta}\left(t_{m}\right)$ is calculated on roughly 6 million configurations, while for the standard action the calculation is carried out on more than 16 million configurations. The shear viscosities increase roughly with $T^{3}$, and $\eta / s$ ratios are close to the KSS lower bound in the region where $1<T / T_{c}<25$. Using these data the bulk viscosities are also determined in the region where $T / T_{c}<2$. They are roughly one order of magnitude smaller than the shear viscosities. Our next target is to determine the transport coefficients more precisely by a maximum-entropy method. For this purpose the most effective method may be to adopt an anisotropic lattice. In this report, we study the possible systematic error due to the deformation of the anisotropic lattice at short distances. Near the critical temperature, it is found that the standard action suffers from a large deformation on the anisotropic lattice at short distances, while the deformation is slight for Iwasaki's improved action. To reduce the fluctuation of the Matsubara Green function, the improvement of the energy momentum tensor operator by using clover-type loops is promising. We are also attempting to apply the multi-level algorithm to reduce fluctuation.

The XXV International Symposium on Lattice Field Theory

30 July-4 August 2007

Regensburg, Germany

\footnotetext{
${ }^{*}$ Speaker.
} 


\section{Introduction and formalism}

A new state of matter has been reported in RHIC experiments. From an estimation of the temperature, it is expected that quark gluon plasma (QGP) is realized there. A phenomenological study of elliptic fbw revealed that a new state of matter is well explained by the fluid with very small viscosity. Jet quenching data indicate that it is strongly interacting.

If QGP is realized as a fluid, its viscosities should be smaller than those of usual fluids such as water and oil, because they are proportional to $g^{-2}$, and the coupling constant $g$ is larger than $1 / 137$ even above the transition temperature. The lower bound for the ratio of shear viscosity $(\eta)$ to entropy(s) has recently been conjectured by Kovtun et al.[1]. However, values close to transition temperature should be calculated from QCD by fully taking into account the nonperturbative effects. It is urgently necessary to carry out detailed phenomenological studies of the new state of matter using these viscosities.

The transport coeffi cients are calculated in the framework of linear response theory:

$$
\begin{gathered}
\eta=-\int d^{3} x^{\prime} \int_{-\infty}^{t} S t_{1} e^{\varepsilon\left(t_{1}-t\right)} \int_{-\infty}^{t_{1}} d t^{\prime}<T_{12}(\vec{x}, t) T_{12}\left(\overrightarrow{x^{\prime}}, t^{\prime}\right)>_{r e t} \\
\frac{4}{3} \eta+\zeta=-\int d^{3} x^{\prime} \int_{-\infty}^{t} d t_{1} e^{\varepsilon\left(t_{1}-t\right)} \int_{-\infty}^{t_{1}} d t^{\prime}<T_{11}(\vec{x}, t) T_{11}\left(\vec{x}^{\prime}, t^{\prime}\right)>_{r e t}
\end{gathered}
$$

where $\eta$ is shear viscosity, and $\zeta$ is bulk viscosity. $\left\langle T_{\mu \nu} T_{\rho \sigma}>_{\text {ret }}\right.$ is the retarded Green function of the energy momentum tensor. In pure gauge theory it is given by

$$
T_{\mu v}=2 \operatorname{Tr}\left[F_{\mu \sigma} F_{v \sigma}-\frac{1}{4} \delta_{\mu v} F_{\rho \sigma} F_{\rho \sigma}\right]
$$

and the fi eld strength tensor $F_{\mu \nu}$ is defi ned by the plaquette operator on a lattice: $U_{\mu v}(x)=\exp \left(i a^{2} g F_{\mu v}(x)\right)$.

The transport coeffi cients are also expressed by the slope of the spectral function $\rho(\omega)$ at $\omega=0$ of the corresponding retarded Green function. The shear viscosity $\eta$ is written as

$$
\eta=\pi \lim _{\omega \rightarrow 0} \frac{\rho(\omega)}{\omega} .
$$

On a lattice, we determine $\rho(\omega)$ by the Matsubara Green function $G_{\beta}$ instead of the retarded Green functions themselves, because both Green functions have the same spectral function,

$$
G_{\beta}\left(t_{m}\right)=-\frac{1}{\beta} \sum_{n} e^{-i \omega_{n} t_{m}} \int_{-\infty}^{\infty} \frac{\rho(\omega)}{i \omega_{n}-\omega} d \omega=\int_{0}^{\infty} \frac{\cosh \left(\omega\left(t_{m}-\beta / 2\right)\right)}{\sinh (\omega \beta / 2)} \rho(\omega) d \omega,
$$

where $\omega_{n}=2 \pi n / \beta$ for the Matsubara Green function. If $i \omega_{n}$ is replaced by $p_{0}+i \varepsilon$, the retarded Green function is obtained.

However, there are still diffi culties in the determination of $\rho(\omega)$. One is that $G_{\beta}\left(t_{m}\right)$ is discrete, while $\rho(\omega)$ is continuous. Therefore, fi ne resolution in the temperature direction (simulation on large $N_{T}$ lattice) is necessary for its accurate determination. The other diffi culty is that $G_{\beta}(t)$ 


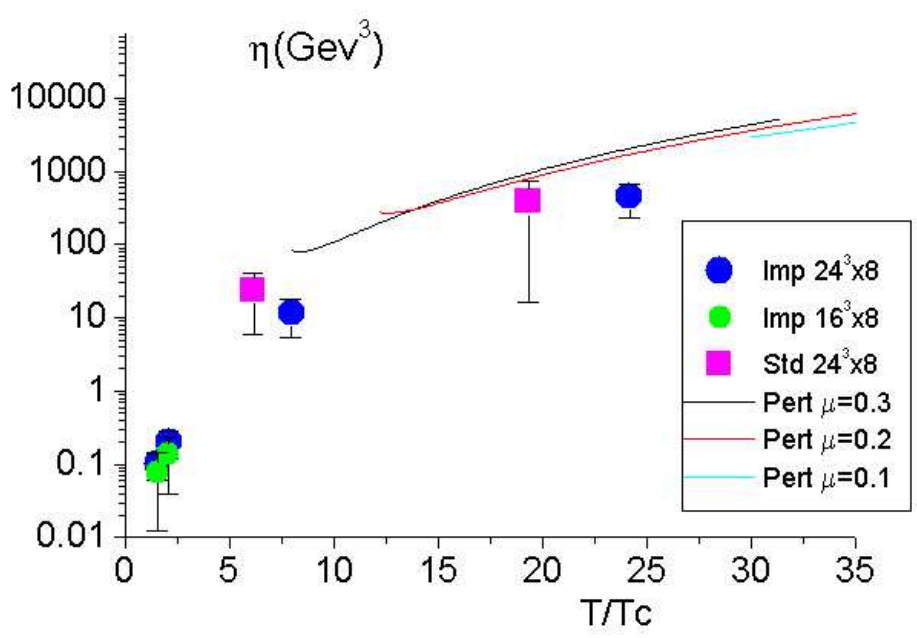

Figure 1: Shear viscosity in physical units from lattice and perturbative calculations. The circles are results from the improved action and squares are those from the standard action. The perturbative results beyond leading log approximations [6] are shown by lines.

is noisy; thus its determination requires much CPU time. Hence, we start with the smaller $N_{T}$ lattice, assuming a plausible form for the spectral function $\rho(\omega)$ that fi ts $G_{\beta}\left(t_{m}\right)$ well. The simplest nontrivial form is [2],

$$
\rho(\omega)=\frac{A}{\pi}\left(\frac{\gamma}{(m-\omega)^{2}+\gamma^{2}}-\frac{\gamma}{(m+\omega)^{2}+\gamma^{2}}\right)
$$

This form is derived from a perturbative calculation in $\varphi^{4}$ theory[3].

\section{Numerical results from isotropic lattice}

Because $\rho(\omega)$ given by Eq.(1.6) has three free parameters, $G_{\beta}\left(t_{m}\right)$ should be calculated on $N_{T} \geq 8$ latticels. We carry out simulations on $24^{3} \times 8$ and $16^{3} \times 8$ lattices, using Iwasaki's improved action and the standard action, and the temperature range is $1.4<T / T_{c}<25$. We are attempting to overcome the huge fluctuations by a large number of measurements. In the case of the improved action, $G_{\beta}\left(t_{m}\right)$ is determined by roughly 6 million measurements, while for the standard action there are more than 16 million measurements. The fi $\mathrm{t}$ of $G_{\beta}\left(t_{m}\right)$ is made by SALS, and errors are estimated by the jackknife method. Then the viscosities are obtained by the formula $\eta a^{3}=$ $4 A \gamma m /\left(\gamma^{2}+m^{2}\right)^{2}$.

To obtain the viscosities in physical units, we need the lattice spacing $a(g)$. For the improved action, $a$ has been determined for $2.2<\beta<3.8$ by the Tsukuba group[ $[$ ], and for the standard action, $a$ has been determined for $5.58<\beta<6.5$ by Edward et al.[5]. Outside these regions, we assume a two-loop asymptotic scaling relation.

The results for shear viscosity $\eta$ in physical units are shown in Fig.1. Because the $\eta \times a^{3}$ has weak $T$ dependence, the shear viscosity increase roughly with $T^{3}$ throughout the temperature region. We fi nd little difference between the results from $24^{3} \times 8$ and $16^{3} \times 8$ lattices. Thus, the size 


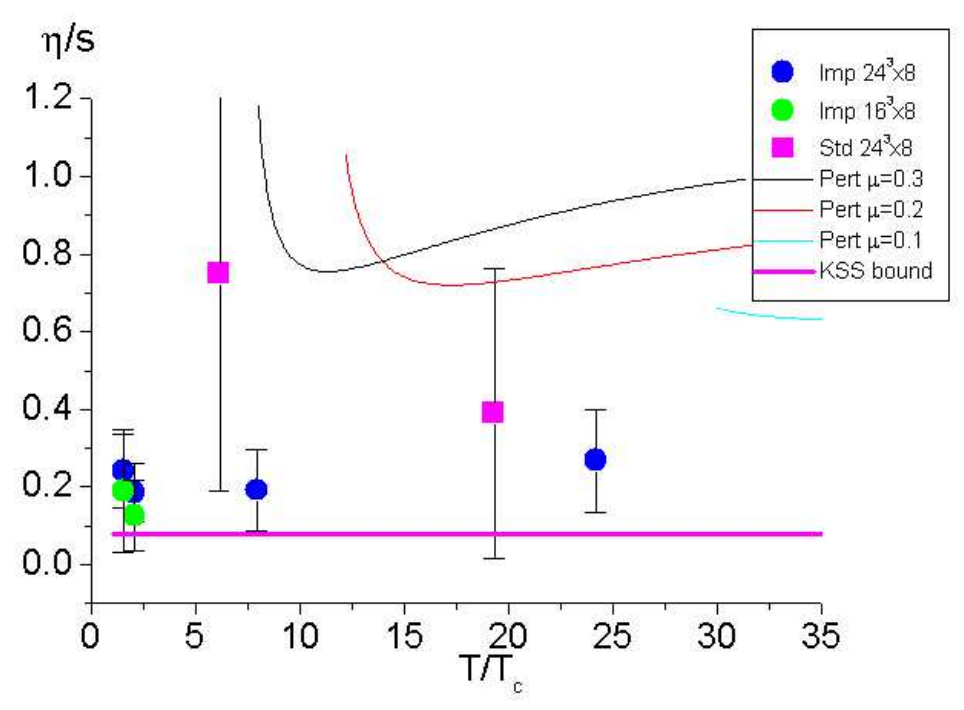

Figure 2: $\eta / s$ obtained by lattice simulations (circles and squares) and perturbative calculations (lines). The KSS bound[1] is also shown.

effect may not be large for the lattices of these sizes. However, more accurate data are necessary to determine quantitative size dependences.

We have also shown the perturbative results beyond the leading log approximation[6] in Fig. 1], where the scale factor $\mu$ in the running coupling constant is a free parameter. The agreement improves when $\mu$ becomes smaller, but in this case the breakdown of the perturbative calculation starts at a higher temperature.

Let us proceed to the $\eta / s$ ratio, recently studied by Kovtun et al.[1]. The results are shown in Fig.2. The lattice data on entropies are given by Ref. [4. 7]. The perturbative results are also shown, where we use the entropy calculated by the hard thermal loop approximation given in Ref.[8]. Because both $\eta a^{3}$ and $s a^{3}$ have weak T dependence in the $T>1.5 T_{c}$ region, the $\eta / s$ ratio also has weak $\mathrm{T}$ dependence and the lattice results are close to the KSS bound for $T / T_{c}<25$.

In Fig. 3 , we show the ratio in RHIC temperature regions together with the bounds by Meyer [9], who employed a multi-level algorithm and got bounds without using an ansatz.

For the improved action, signals for the bulk viscosities begin to overcome the errors, when there are about 6 million measurements. The results are shown in Fig. 4 . The values of bulk viscosity still have rather large errors, but at $\mathrm{T}$ close to $T_{c}$, their values are determined, which are roughly one order of magnitude smaller than the shear viscosities. Their $T$-dependence is an interesting problem but that requires more measurements. Recently it has been claimed that the bulk viscosity is large near the critical temperature and decreases rapidly with $\mathrm{T}[10]$. Our results do not contradict this. In the case of the standard action, the bulk viscosities still have large errors that they cannot be determined.

It will be interesting to carry out phenomenological studies on RHIC data taking into account these viscosities in the fluid model.

\subsection{Discussions}

- The renormalization factor $Z$ of the energy momentum tensor is discussed by Meyer[ק]: 


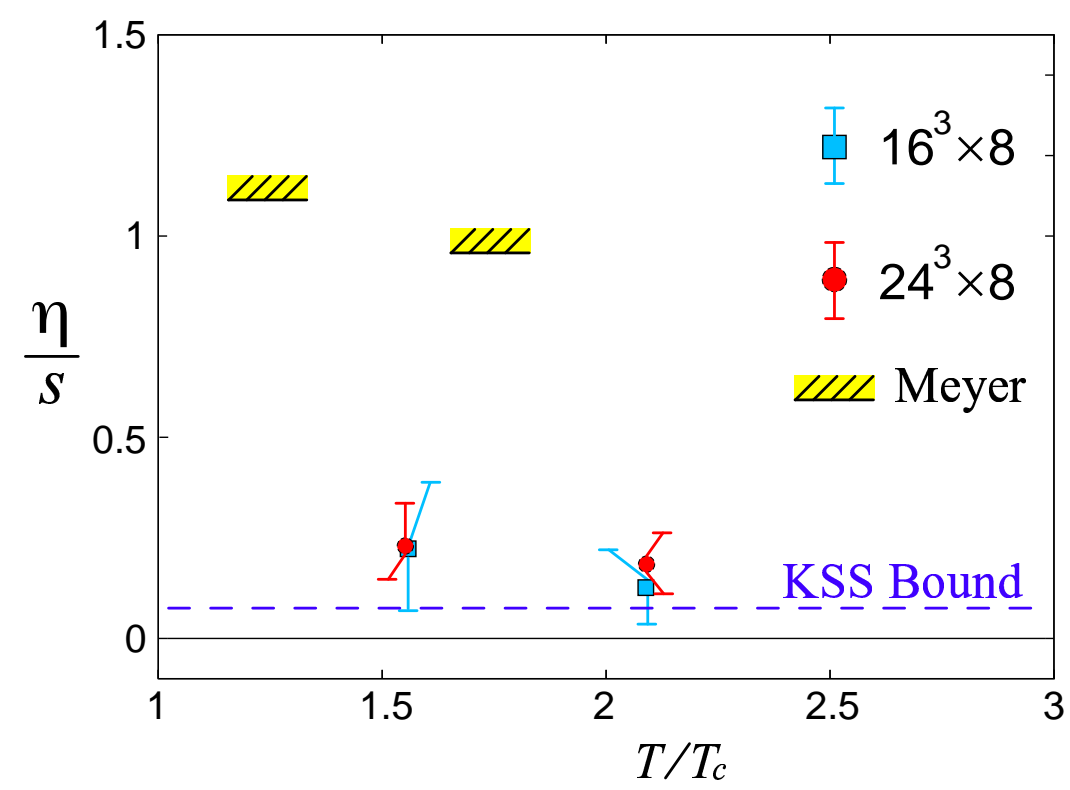

Figure 3: Ratio of $\eta / s$ in RHIC regions.

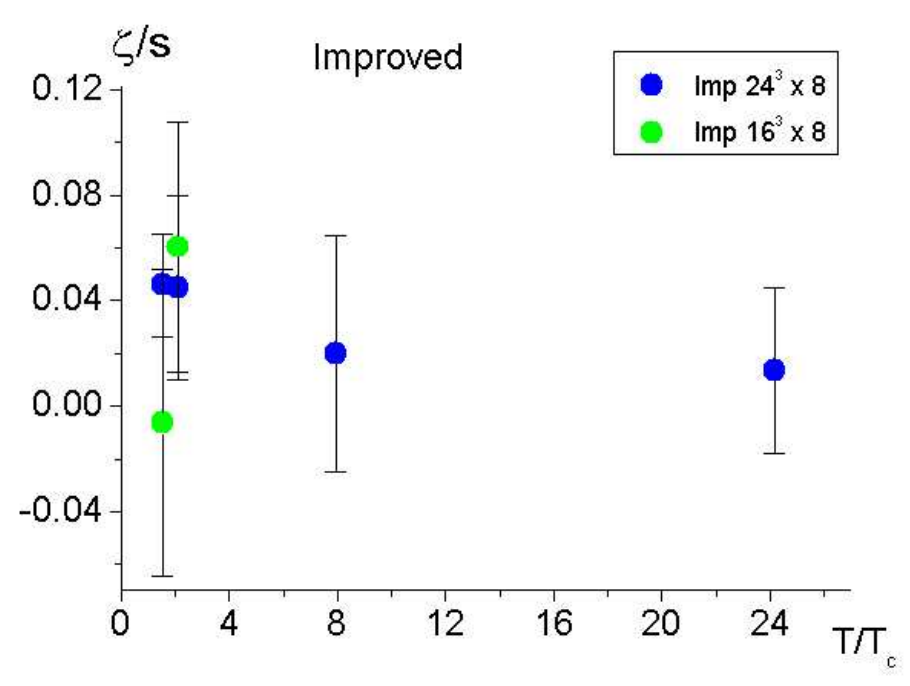

Figure 4: Lattice results of $\zeta / s$ ratio from the improved action

$Z=1-g^{2} / 2\left(c_{\sigma}-c_{\tau}\right)$. If the parametrization of $\mathrm{Z}$ factor given by Ref.[阿] is used, the viscosities calculated by the standard action decrease by about $30 \%$. Z factor can also be written as follows: $Z=\partial \gamma / \partial \xi[14$, where $\xi$ is the renormalized anisotropy and $\gamma$ is the bare anisotropy. In the case of Iwasaki's improved action, $\xi \sim \gamma$ over a wide range of $\beta$ and $\xi$; therefore the $\mathrm{Z}$ factor is close to 1 . If the $\mathrm{Z}$ factor is taken into account, the difference between $\eta$ obtained from improved action and standard action decreases.

- We have attempted to fi t $G_{\beta}^{2}$ by other parametrizations of $\rho(\omega)$ than that given in Eq.1.6. If we apply the formula for $\rho$ proposed in Ref.[11], the fi t is not satisfactory and $\rho$ does not satisfy the constraint $\omega \rho(\omega)>0[12$ ]. If we truncate the Taylor expansion of $\rho(\omega)$ after the lowest 3 

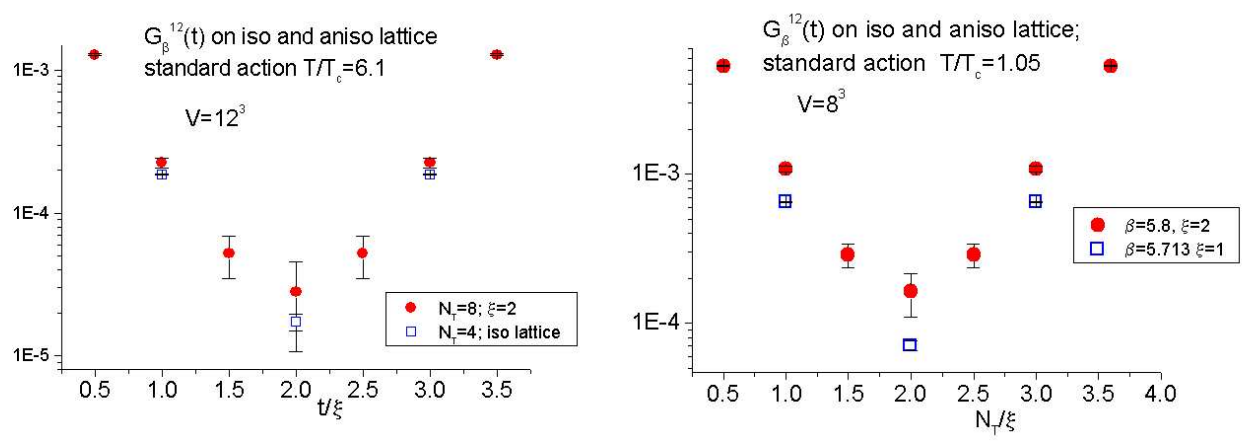

Figure 5: $G_{\beta}^{12}\left(t_{m}\right)$ on isotropic $\left(N_{T}=4\right)$ and $\xi=2$ anisotropic lattice $\left(N_{T}=8\right)$

terms, the fi $t$ is also not satisfactory and the coeffi cient of $\omega$ becomes negative, which also cannot be accepted as a spectral function. In the three-parameter functions for $\rho$, we have not found a $\rho(\omega)$ that fi ts $G_{\beta}^{2}\left(t_{m}\right)$ well except for Eq.1.6.

\section{Next project: toward the high-precision calculation of $\eta$}

\subsection{Simulation on anisotropic lattice}

To determine $\rho(\omega)$ without relying on any assumption, the maximum entropy method(MEM) seems promising. To get a reliable result, accurate $G_{\beta}$ for a $N_{T} \sim 30$ lattice is necessary. For this purpose, the best method may be to adopt an anisotropic lattice. Before carrying out the simulation on large anisotropic lattices, we start with a preliminary study on the possible systematic error.

The fundamental properties of anisotropic lattices have already been studied[13, 14, 15]. The anisotropy $\xi=a_{\sigma} / a_{\tau}$ is controlled by the bare anisotropy $\gamma$ in the action. The $\gamma$ dependence of $\xi$ is expressed in terms of the ratio $\xi / \gamma[13,14,15]$. The $\xi / \gamma$ ratio is determined by its asymptotic plateau for $r \geq 3$, where $\mathrm{r}$ is the distance of the lattice in the unit of space direction $a_{\sigma}$. At short distances, the $\xi / \gamma$ ratio is not equal to its asymptotic value. Thus $G_{\beta}\left(t_{m}\right)$ for small $t_{m} / \xi$ will suffer from a systematic error due to deformation.

To study the effects of the deformation, we compare $G_{\beta}^{12}$ for isotropic and $\xi=2$ anisotropic lattices at the same $T / T_{c}\left(a_{\sigma}\right)$. For the standard action, $G_{\beta}^{12}$ is shown in Fig.5. Large discrepancies are observed near $T_{c}$, due to the deformation at short distances $\left(t_{m} / \xi \leq 2\right)$ and to the difference between the $\mathrm{Z}$ factor in the lattices. An increase in the deformation is anticipated as the $\xi / \gamma$ ratio increases toward $T_{c}$. On the other hand in the case of improved action, the difference in $G_{\beta}^{12}\left(t_{m}\right)$ for the lattices is small. In this action, because the ratio $\xi / \gamma$ is close to unity over a wide range of $\beta$ and $\xi$, the deformation is weak and the $\mathrm{Z}$ factor is close to unity.

\subsection{Improvement of the energy momentum operator}

To reduce the fluctuation of $G_{\beta}$, we have attempted to use clover-type operator for the defi nition of the energy momentum tensor. It was found that the noise is strongly suppressed, and the normalization and the $t$-dependence of $G_{\mu v}(t)$ are not changed. We are also testing the effectiveness of the multi-level algorithm[16] for reducing the fluctuations[ 7$]$. 


\section{Conclusion}

The shear and bulk viscosities are calculated on isotropic $24^{3} \times 8$ and $16^{3} \times 8$ lattices. The T-dependence of the $\eta / s$ ratio is weak and its value is close to KSS bound throughout the region $T / T_{c}<25$. The bulk viscosities are obtained in the $T / T_{c} \leq 2$ region, and their values are one order of magnitude smaller than those of shear viscosities.

The systematic error due to the deformation of lattice spacing at a short distance is studied. For the standard action, the deformation becomes strong near $T_{c}$. In the $t_{m} / \xi \leq 3$ region, the effects of deformation should be carefully controlled.

For the accurate determination of the spectral function $\rho$ by MEM, improvements of the energy momentum tensor operator using clover-type loops and the multi-level algorithm are promising.

\section{Acknowledgments}

We thank R. Gupta, Y. Koma and T. Umeda for helpful discussions. The simulations were carried out on SX5 and SX8 at RCNP. We are grateful to the members of RCNP for their kind support. This work was supported by Grants-in-Aid for Scientifi c Research from Monbu-Kagakusho (The Ministry of Education, Culture, Sports, Science and Technology) (No. 17340080).

\section{References}

[1] G. Policastro, D.T. Son and A.O. Starinet, Phys. Rev. Lett. 87 (2001) 081601. P.K. Kovtun, D.T. Son and A.O. Starinet, Phys. Rev. Lett. 94 (2005) 111601.

[2] F. Karsch and W. Wyld, Phys. Rev. D35 (1987) 2518.

[3] A. Hosoya, M. Sakagami and M. Takao, Anal. Phys. 154 (1984) 229.

[4] M. Okamoto et al., Phys.Rev. D60 (1999) 094510.

[5] R.G. Edward, U. M. Heller and T. R. Klassen, Nucl. Phys. B517 (1998) 377.

[6] P. Arnold, G.D. Moore and L.G. Yaffe, JHEP 0305 (2003) 051.

[7] G. Boyd et al., Nucl. Phys. B469 (1996) 419.

[8] J.-P. Blaizot, E. Iancu and A. Rebhan, Phys. Rev. Lett. 83 (1999) 2906.

[9] H. Meyer, A calculation of the shear viscosity in SU(3) gluodynamics [hep-lat/0704.1801vl].

[10] D. Kharzeev and K. Tuchin, Bulk viscosity of QCD matter near critical temperature [hep-ph/0705.4280v2].

[11] G. Aarts and J.M.M. Resco, JHEP 0204 (2002) 053.

[12] M. Le Bellac, Thermal Field Theory, Cambridge University Press, 1996.

[13] T.R. Klassen, Nucl. Phys. B533 (1988) 557.

[14] J. Engels, F. Karsch and T. Scheideler, Nucl. Phys. B 567 (2000) 303.

[15] S. Sakai, T. Saito and A. Nakamura, Nucl. Phys. $B 584$ (2000) 528. S. Sakai and A. Nakamura, Phys. Rev. D69 (2004) 114504.

[16] M. L and P. Weise, JHEP 0109 (2001) 010.

[17] R. Gupta, M. Koma, Y. Koma, A. Nakamura, S. Sakai and T. Umeda, work in progress. 